\title{
Improving Ad Hoc Networks Capacity and Connectivity Using Dynamic Blind Beamforming
}

\author{
Nadia Fawaz, Zafer Beyaztas, David Gesbert \\ Mobile Communications Department, Eurecom Institute \\ Sophia-Antipolis, France \\ \{fawaz, beyaztas, gesbert $\} @$ eurecom.fr
}

\begin{abstract}
We propose a dynamic blind beamforming scheme which allows to benefit from antenna directivity in large mobile ad hoc networks while avoiding heavy feedback to track mobile nodes localization. By orienting its directional antenna successively in all directions, a source surely but blindly hits its destination without knowing its exact position. Performance is analyzed in terms of total network throughput and connectivity and the optimal number of rotations allowing to maximize performance is shown to result from a trade-off between delay and improvements in terms of interference. In large ad hoc networks, known to be interference limited, we show that dynamic blind beamforming can outperform omnidirectional transmissions both in terms of capacity and connectivity.
\end{abstract}

\section{INTRODUCTION}

In ad hoc networks, in particular large thus interferencelimited ones, directional antennas improve performance when the destination position is known [1]-[5]. Nevertheless in high-mobility context, the feedback required for localization tracking increases the transmission protocol overhead [6]-[8] and thus reduces the useful rate leading to consider mainly omnidirectional antennas. The question we address is how to benefit from directional antennas or beamforming while avoiding the feedback load especially in large ad hoc networks.

\section{A. The Idea In Brief}

We propose a dynamic blind beamforming scheme which allows to benefit from antenna directivity in large mobile ad hoc networks while avoiding heavy feedback usually due to mobility and density. The scheme is dynamic and blind since a source uses a rotating antenna successively in all directions to surely but blindly hit its destination without knowing its exact position. If position is known with a certain accuracy, for example thanks to limited feedback, a source can beamform semi-blindly in a subset of directions.

Rotational directivity has a major impact on interference and thus on capacity: by focusing the transmitted power successively in different directions, the probability of interfering with other destinations, i.e. hitting a non-intended destination at the same time it is receiving a signal from its own source, is low because of both spatial focusing and asynchronism of all communications. Nevertheless when rotating the antenna, some time is wasted when the source is not beamforming in the direction of its intended destination. These two opposite effects lead to a capacity-delay trade-off when tuning the number of rotations.

\author{
Merouane Debbah \\ Alcatel-Lucent Chair on Flexible Radio, SUPELEC \\ Gif-sur-Yvette, France \\ merouane.debbah@supelec.fr
}

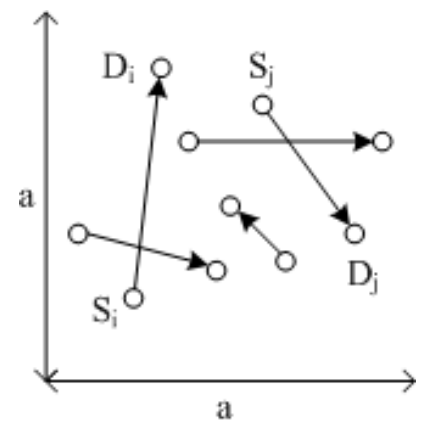

Fig. 1. 2D-Ad Hoc Network

We analyze performance in terms of total network throughput and connectivity and show that our scheme can outperform omnidirectional transmissions in ad hoc networks and that depending on the density of the network, an optimal number of rotations allows to maximize the network performance. In the final version of this paper we intend to also include curves for the ideal directional case where all positions are known perfectly at any time thanks to constant and full feedback, as an upper bound for the dynamic blind beamforming case.

\section{B. Related Work}

Recently Sharif and Hassibi [9] proposed a random beamforming scheme for the Multi-user MIMO Broadcast channel in which the transmitter constructs random beams and transmits to the users with the highest SINRs, fedback to the transmitter. When the number of users increases, the capacity was shown to scale as with perfect CSI at the transmitter. Nevertheless this random beamforming model relies on feedbacks from mobile units to a BTS in a cellular system and the served-destinations are chosen according to the quality of their link to the BTS for a given set of random beams.

On the other hand Bettstetter et al. [10] showed that random beamforming in ad hoc networks can improve received-powerconnectivity: sources send random beams in a random direction they chose once and for all at the beginning; any node whose received power from a source is above a threshold is considered connected to the source. No source and destination are associated in a communicating pair idea, i.e. a source ignores not only the position but also the identity of the destinations. Anyone who hits a receiver strongly enough is 
connected to him. What about interferences if two sources hit a destination at the same time? The received-power-connectivity criterion does not take into account interferences as an SINR criterion would do, nor reliable decoding issues that are usually illustrated by capacity or BER.

\section{Outline}

The rest of the paper is organized as follows. In section II, notations and the system model are presented. In section III the performance criteria are described. Numerical results and comparisons with the omnidirectional scenario are provided in section IV and lead to the concluding section $\mathrm{V}$.

\section{SYSTEM MODEL}

Consider the 2D-network of $M$ communicating pairs $\left\{S_{i} D_{i}\right\}_{i \in\{1, \ldots, M\}}$ uniformly distributed over a square area $a^{2} m^{2}$, illustrated in figure 1. $d_{j i}=S_{i} D_{j}$ denotes the distance between source $S_{i}$ and destination $D_{j}$. All nodes are equipped with a single antenna, directional at sources and omnidirectional at destinations.

Each source $S_{i}$ generates a sequence $s_{i}(n), n \in$ $\left\{0, \ldots, N_{s}-1\right\}$. These symbols are modeled by independent identically distributed (i.i.d.) circularly-symmetric complex gaussian random variables with zero mean. At time $t=k T$, the signal transmitted by source $S_{i}$ is denoted $x_{i}(k)$ whereas $y_{j}(k)$ represents the signal received by destination $D_{j}$.

Sources have the ability to rotate their directional antenna, selecting a different transmission direction at each time-slot $T$. $N$ denotes the number of times a source rotates its directional antenna to transmit the same symbol repetitively in $N$ timeslots, pointing at a different direction during each time slot with a beamwidth $\alpha=\frac{2 \pi}{N} . N=1$ corresponds to the case where source antennas are omnidirectional. After $N$ timeslots, the source has sent the same symbol $N$ times in $\mathrm{N}$ successive directions, covering the whole $2 \pi$-space, like a lighthouse operating in a discrete fashion. For an arbitrary $N$, the signal transmitted by source $S_{i}$ at $k^{\text {th }}$ time-slot is thus: $x_{i}(k)=s_{i}\left(\left\lfloor\frac{k}{N}\right\rfloor\right)$.

The channel between transmitter $S_{i}$ and receiver $D_{j}$ is represented by $h_{j i}$ which includes the effects of shadowing and slow flat fading. These channel coefficients are modeled by independent circularly-symmetric complex gaussian random variables with zero mean and variance $\sigma_{j i}^{2}$, i.e. Rayleigh fading. $z_{j}(k)$ are i.i.d circularly-symmetric complex gaussian noises at receivers, with variance $\sigma^{2}$.

\section{A. Interferers Groups}

Consider the communicating pair $S_{i} D_{i}$. For each symbol $s_{i}(n)$ that $S_{i}$ transmits $N$ times, $D_{i}$ receives the symbol only once, during the time-slot when $D_{i}$ is in the rotating beam of $S_{i}$. Any other source whose beam would cover $D_{i}$ during the time-slot where $D_{i}$ receives a signal from $S_{i}$ belongs to the group $\mathcal{I}_{i}$ of interferers of $D_{i}$. A source whose beam would cover $D_{i}$ when $D_{i}$ is not receiving any signal from $S_{i}$ is not an interferer.

In the omnidirectional case $N=1$, all other sources are interferers, therefore $\forall i \in\{1, \ldots, M\}, \mathcal{I}_{i}=\left\{S_{j} / j \neq i\right\}$ contains $M-1$ interferers. When $N>1$, rotating the

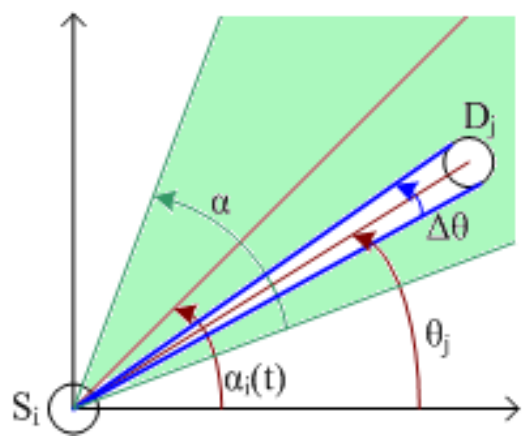

Fig. 2. Transmission between $S_{i}$ and $D_{j}$

antennas clearly allows to decrease the number of interferers per destination with respect to the omnidirectional case and the group of interferers of a destination depends on the network topology and the initial transmission direction chosen by each source.

When $D_{i}$ receives a signal that does not contain any component coming from $S_{i}$, recognized for example thanks to an embedded signature identifying $S_{i}, D_{i}$ simply discards the received signal. One could argue that in a static network, when $D_{i}$ recognizes a signal component from $S_{i}$, it could send a feedback to $S_{i}$ which would then identify the direction in which to beamform. But in a high mobility context, tracking $D_{i}$ moving position would lead to heavy overhead, which our blind dynamic beamforming strategy intends to avoid.

When $D_{i}$ is in the transmission beam of $S_{i}$ at time $t=k T$, the received signal $y_{i}(k)$ at $D_{i}$ is the sum of the signals transmitted by $S_{i}$ and all sources in $\mathcal{I}_{i}$ filtered by their respective channels, and noise $z_{i}(k)$.

\section{B. Transmitted Power and Energy}

We use the simple ideally-sectorized directional antenna model to describe the gain pattern, described in [10] equation (4). As illustrated in figure 2 , it is assumed that at time $t$, the transmit antenna of $S_{i}$ forms a beam of width $\alpha$ in the direction $\alpha_{i}(t)$ with a certain gain. $\alpha_{i}(0)$ denotes the initial direction chosen at random by $S_{i}$ during the first time-slot, then every time-slot, $S_{i}$ rotates its antenna anti-clockwise of an angle $\alpha$ to get the new direction. Thus $\alpha_{i}(t)=\alpha_{i}(0)+\left\lfloor\frac{t}{T}\right\rfloor \alpha$.

Each source has a power constraint in the continuous timechannel of $P_{0}$ Joules/s. In the omnidirectional case, $P_{0}$ is transmitted over the whole $2 \pi$ space with an angular density of power $P_{0} / 2 \pi$, whereas in the directional case $N>1, P_{0}$ is focused in an angle $\alpha=2 \pi / N$ leading to the Power Angular Density at time $t$ in direction $\theta$ :

$$
\frac{\partial P_{i}}{\partial \theta}(\theta, t)=\frac{P_{0}}{\alpha} \mathbb{1}_{\left[\alpha_{i}(t)-\alpha / 2 ; \alpha_{i}(t)+\alpha / 2[\right.}(\theta)
$$

Indeed since a source transmits only in $(1 / N)^{t h}$ of the space, it can increase its transmit power in its transmission beam to $P_{0} / \alpha=N P_{0} / 2 \pi$ and remain within its average power constraint for the whole space. The Power Angular Density depends on time since the transmit antenna rotates. On the contrary, the Total Transmitted Power by source $S_{i}$ does not 
depend on time, nor on the number of rotations $N$ and respects the power constraint by definition:

$$
P_{i}=\int_{\theta=0}^{2 \pi} \frac{\partial P_{i}}{\partial \theta}(\theta, t) \partial \theta=\int_{\theta=\alpha_{i}(t)-\alpha / 2}^{\alpha_{i}(t)+\alpha / 2} \frac{P_{0}}{\alpha} \partial \theta=P_{0}
$$

The transmitted power is by definition $P_{i}=\frac{\partial \varepsilon_{i}}{\partial t}$, where $\varepsilon_{i}$ is the energy transmitted by $S_{i}$. Since source $S_{i}$ transmits the same symbol in $N$ time-slots, the total transmitted energy for symbol $s_{i}(n)$ is :

$$
\varepsilon_{i}=\int_{t=n N T}^{(n+1) N T} P_{i} \partial t=N T P_{0}
$$

Thus in the rotational directional case $N>1$, the transmitted energy is $N$ times greater than in the omnidirectional case, but only part of the transmitted energy will be collected at the destination, during the single time-slot where the destination is in the transmission beam.

\section{Received Power and Energy}

The effective aperture of the omnidirectional antenna at a destination is an area $A_{e}$ and the associated angular aperture is $\Delta \theta$, whereas $\theta_{j}$ represents $D_{j}$ angular position in polar coordinates in the plane as in figure 2 . We assume that the effective aperture $A_{e}$ is small with respect to distances between nodes, so that the variations of the angular aperture with the position of the node can be neglected.

The received power $P_{j i}(t)$ at $D_{j}$ coming from $S_{i}$ depends on time, since the destination needs to be in the rotating beam to receive power from $S_{i}$.

$$
\begin{aligned}
P_{j i}(t) & =\frac{\left|h_{j i}\right|^{2}}{d_{j i}^{2}} \int_{\theta=\theta_{j}-\frac{\Delta \theta}{2}}^{\theta_{j}+\frac{\Delta \theta}{2}} \frac{\partial P_{i}}{\partial \theta}(\theta, t) \partial \theta \\
& =\frac{\left|h_{j i}\right|^{2} P_{0}}{d_{j i}^{2} \alpha} \int_{\theta=\theta_{j}-\frac{\Delta \theta}{2}}^{\theta_{j}+\frac{\Delta \theta}{2}} \mathbb{1}_{\left[\alpha_{i}(t)-\alpha / 2 ; \alpha_{i}(t)+\alpha / 2[\right.}(\theta) \partial \theta \\
& = \begin{cases}\frac{\left|h_{j i}\right|^{2}}{d_{j i}^{2}} \frac{P_{0} \Delta \theta}{\alpha} & \text { if } \theta_{j} \text { is in } S_{i} \text { beam at time } t \\
0 & \text { otherwise }\end{cases}
\end{aligned}
$$

where $\frac{\Delta \theta}{\alpha}$ represents the fraction of power that the destination receives from the beam of width $\alpha$, due to the finite size of the receive antenna.

$S_{i}$ transmits energy $\varepsilon_{i}$ for symbol $s_{i}(n)$ between $t=n N T$ and $t=(n+1) N T$, but $D_{j}$ receives energy $\varepsilon_{j i}$ for symbol $s_{i}(n)$ only during the time-slot $T$ when $S_{i}$ beamforms in the direction of $D_{j}$, leading to the expression:

$$
\varepsilon_{j i}=\int_{t=n N T}^{(n+1) N T} P_{j i}(t) \partial t=\frac{P_{0}\left|h_{j i}\right|^{2} \Delta \theta}{d_{j i}^{2} \alpha} T=N \varepsilon_{j i}^{o m n i}
$$

where $\varepsilon_{j i}^{o m n i}=\frac{P_{0}\left|h_{j i}\right|^{2} \Delta \theta}{d_{j i}^{2} * 2 \pi} T$ is the energy received by $D_{j}$ for a symbol $s_{i}(n)$ transmitted during only one time-slot in the omnidirectional case. When $N>1$ the received energy $\varepsilon_{j i}$ for symbol $s_{i}(n)$ at $D_{j}$ is $N$ times greater than in the omnidirectional case because of the spatial focusing effect of the directional transmit antenna. Without a loss of generality, we will consider that $T=1$ and simplify expressions.

\section{PERFORMANCE ANALYSIS}

In this section, we derive the performance criteria to compare the dynamic blind beamforming strategy to the omnidirectional transmission in terms of total network throughput and throughput-based connectivity.

\section{A. Total Network Throughput}

For a communicating pair $S_{i} D_{i}$, the mutual information [11] between input $s_{i}$ and output $y_{i}$ at $D_{i}$, according to (5), is given by:

$$
\begin{aligned}
I\left(s_{i} ; y_{i}\right) & =\log \left(1+\frac{\varepsilon_{i i}}{\sigma^{2}+\sum_{j \in \mathcal{I}_{i}} \varepsilon_{i j}}\right) \\
& =\log \left(1+\frac{\rho N \frac{\left|h_{i i}\right|^{2} \Delta \theta}{2 \pi d_{i i}^{2}}}{1+\rho \sum_{j \in \mathcal{I}_{i}} N \frac{\left|h_{i j}\right|^{2} \Delta \theta}{2 \pi d_{i j}^{2}}}\right)
\end{aligned}
$$

where the input SNR is $\rho=P_{0} / \sigma^{2}$. Since the source hits its intended destination only once in $N$ successive rotational trials, the throughput of user $i$ is given by

$$
C_{i}=\frac{1}{N} I\left(s_{i} ; y_{i}\right)
$$

where the factor $1 / N$ in front of the log accounts for the waste of time in the transmission of a symbol.

The total network throughput is given by:

$$
C=\frac{1}{N} \sum_{i=1}^{M} \log \left(1+\frac{\rho N \frac{\left|h_{i i}\right|^{2} \Delta \theta}{2 \pi d_{i i}^{2}}}{1+\rho N \sum_{j \in \mathcal{I}_{i}} \frac{\left|h_{i j}\right|^{2} \Delta \theta}{2 \pi d_{i j}^{2}}}\right)
$$

As previously mentioned, the use of rotating directional antennas allows to decrease the number of interferers in a group $\mathcal{I}_{i}$ and to focus the power in a direction, increasing the received power at the destination. But the spatial focusing also makes an interferer hit a non-intended destination stronger than in the omnidirectional case. The greater $\mathrm{N}$, the narrower the beam thus the smaller the number of interferers and the higher the useful received power, but also the stronger the power of interference and the greater waste of time, suggesting a trade-off. The positive impact of the dynamic blind beamforming on the network throughput might not look obvious a priori, but it is shown in section IV.

\section{B. Throughput-based Connectivity}

Several definitions of connectivity exist, they have in common that two nodes are said to be connected if some criterion is above a threshold. In [10], connectivity is defined with respect to the level of received power, but this definition does not take into account interference. To take into account interference, an SINR-based definition of connectivity can be considered. Nevertheless in the case of the dynamic blind beamforming technic we propose, defining the connectivity in terms of SINR above a threshold would lead to ignore the waste of time represented by the factor $1 / N$ in the throughput formula. Indeed, it would be as if a pair was said to be always connected with a certain SINR, when the pair is actually discontinuously connected, only once every $N$ time-slots. 


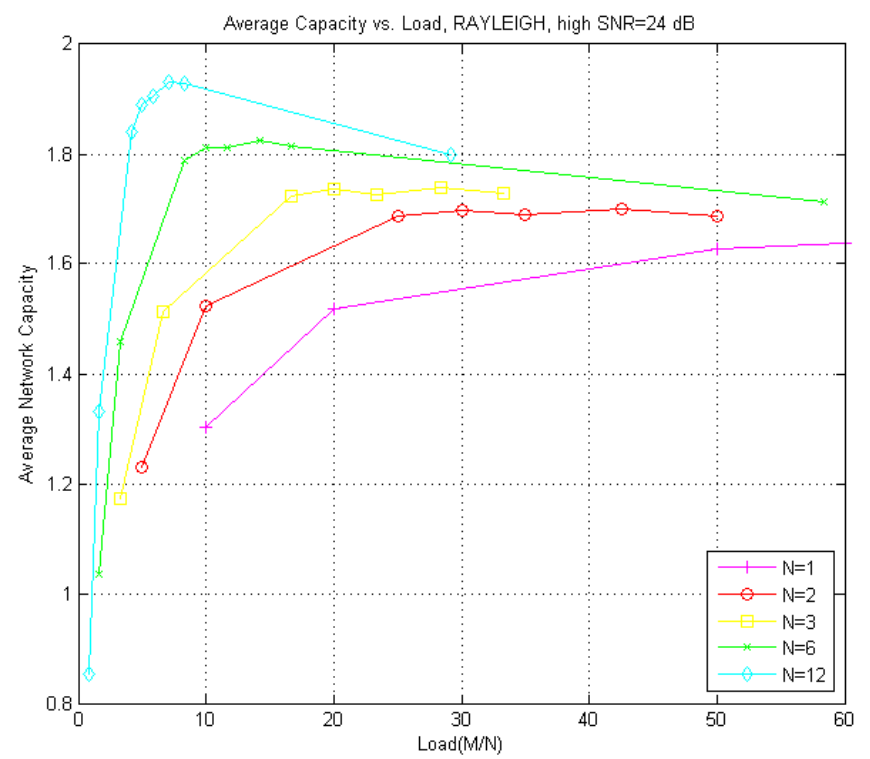

(a) Average Network Capacity versus Load

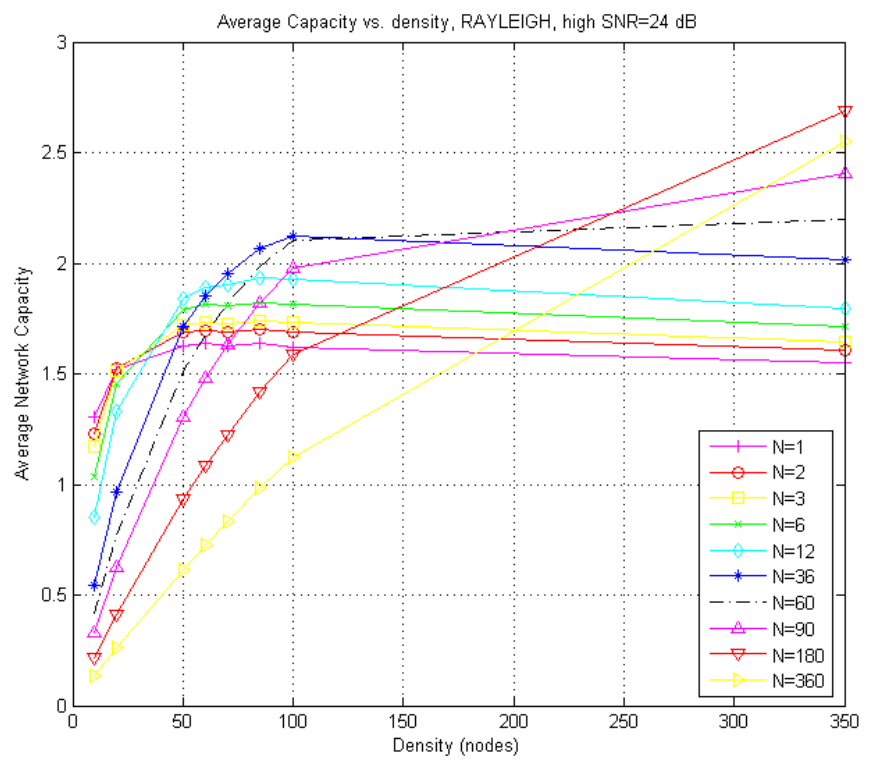

(b) Average Network Capacity versus Density

Fig. 3. Comparison of Network Capacities For Different Rotational Scenarios

The Information-theoretic point of view of connectivity, considering rate as the criterion to define connectivity, appears to be a more relevant and appropriate definition of connectivity. In particular, the notion of rate threshold makes sense in a quality-of-service approach, where users have target rates that need to be satisfied whatever happens. Inspired by [12] and taking into account the factor $1 / N$, we define connectivity with target throughput $\mathrm{R}$ as follows: "A pair is connected if the source can communicate with its intended destination with a throughput at least R".

The network throughput-based connectivity $\kappa$ is defined as the number of connected pairs divided by the number of pairs in the network, i.e. the proportion of pairs to which a throughput $\mathrm{R}$ can be guaranteed:

$$
\kappa=\frac{\left|\left\{i / I\left(s_{i} ; y_{i}\right) \geq R\right\}\right|}{M}
$$

\section{NUMERICAL RESULTS}

In this section, numerical results are presented to compare the different transmission strategies. Monte-Carlo Simulations of 10,000 different topologies were performed for different values of input SNR $\rho$, load $M / N$, number of pairs of nodes $M$ in the network i.e. density. The edge of the area was $a=$ $100 \mathrm{~m}$ and the case of symmetric networks, i.e. in which the fading variances are identical $\sigma_{j i}^{2}=1$, was considered.

\section{A. Total Network Throughput}

We first analyze how the rotational directivity impacts the network throughput. Figures 3(b), 3(a) and (4) illustrate the total network throughput obtained by averaging the throughput over all generated topologies.

Figure 3(a) shows the evolution of the network throughput when the load increases, for different values of $N$. The network throughput reaches a maximum corresponding to an optimal load, then saturates when the load of the network increases. Nevertheless a high number of rotations $N$ allows to support a higher network throughput for a given load and to reach the saturation level later when the load increases.

Figure 3(b) plots the throughputs versus the density of the network, for different values of $N$ at high SNR. A similar behavior as in 3(a) - maximum then saturation - is observed, but the successive intersections of the curves show that the number of rotations maximizing the network throughput increases progressively when the density increases. Indeed at low densities, interference occurring in the network is low and the impact of dynamic blind beamforming on interference is not high enough to compensate the $1 / N$ factor in front of the $\log$ in expression (8). On the contrary, at higher densities, the network becomes interference limited, omnidirectional transmission is not optimal anymore and the improvements in SINR via reduction of the interference thanks to dynamic blind beamforming are important enough to mitigate the $1 / N$ loss. We would like to point out that although we present graphs at high SNR only, for the sake of conciseness, the same behavior is observed in the case of lower input SNR, except that intersections occur at higher densities.

The gains in total network throughput thanks to dynamic blind beamforming for increasing densities are clearly illustrated in figure 4, plotting the network throughput versus the number of rotations $N$, each curve representing a density $\delta$. The curves at the bottom represent low densities, and the curves move toward the top of the graph when density increases. Clearly there exists an optimal $N$ which maximizes the network throughput for each density, illustrating the tradeoff between interference reduction and delay. Using the optimal $N$ allows to dramatically improve the network sum-rate, from $30 \%$ at $M=60$ pairs, up to $70 \%$ at very high densities $(\mathrm{M}=350)$ with respect to omnidirectional transmissions. As 


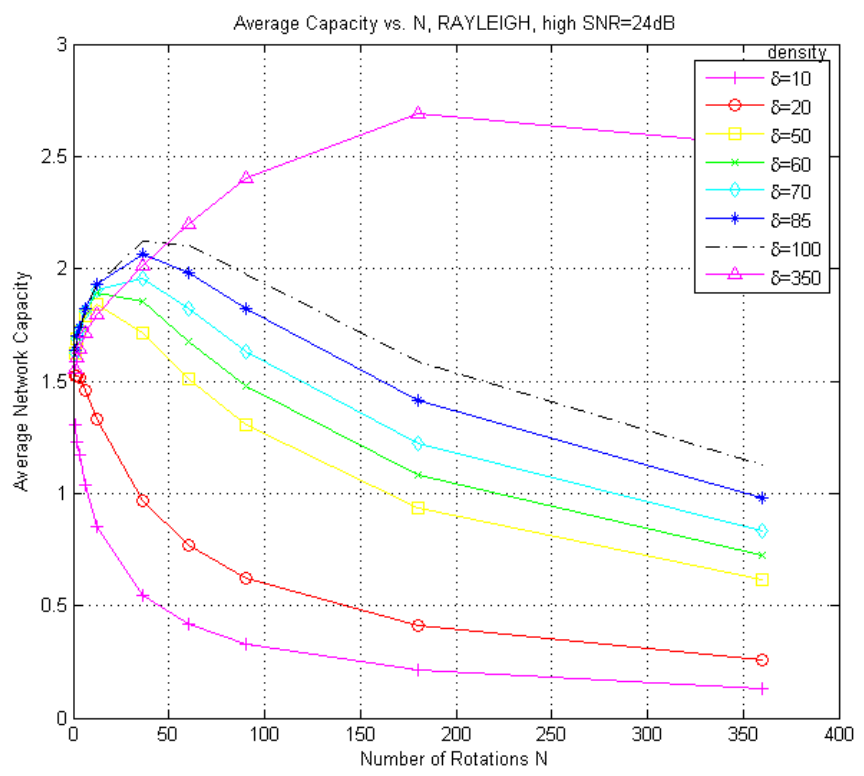

Fig. 4. Average Network Capacity versus N

the density of the network increases, the optimal $N$ increases indicating that beams need to get narrower, but not too quickly so that improvements in terms of interference are not done at the expense of an infinite delay.

In large networks, omnidirectional transmissions are not optimal, and the use of directional antennas even blindly and dynamically allows to enhance the network performance.

\section{B. Network Connectivity}

In terms of network connectivity, a trade-off is illustrated by figure 5 , which plots the average connectivity versus the density for different $N$. Indeed curves can be grouped in two sets : for $2 \leq N \leq 12$ connectivity curves are above the omnidirectional case $N=1$ and for $N>36$ curves are below. When $N$ increases, connectivity is increased up to a certain point, then increasing $N$ decreases connectivity. Network connectivity can thus be improved thanks to dynamic blind beamforming.

\section{CONCLUSION}

We proposed a dynamic blind beamforming technic to benefit from directional antennas while avoiding the feedback load in large ad hoc networks. We analyzed performance in terms of total network throughput and throughput-connectivity and showed that our scheme can outperform omnidirectional transmissions in ad hoc networks. Depending on the density of the network, an optimal number of rotations allows to maximize the network performance. This optimal number of rotations results from a trade-off between introduction of delay and reduction of interference. In large ad hoc networks, which are known to be interference limited, omnidirectional transmissions are not optimal and the use of directional antennas even blindly and dynamically allows to fight against interference and to enhance the network performance. Future work may include analysis of the impact of limited feedback of the

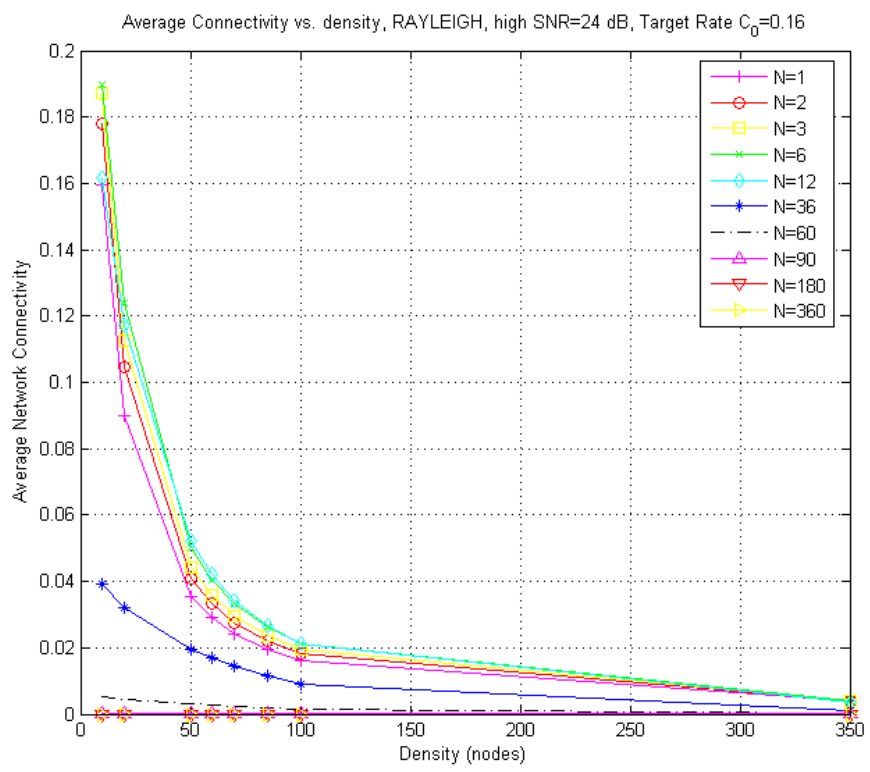

Fig. 5. Average Network Connectivity versus Density

positions on the performance dynamic blind beamforming in particular in a high mobility environment.

\section{ACKNOWLEDGMENT}

This work was supported by the French Defense Body, DGA and by Alcatel-Lucent within the Alcatel-Lucent Chair on flexible radio at SUPELEC.

\section{REFERENCES}

[1] A. Spyropoulos and C. Raghavendra, "Capacity bounds for ad-hoc networks using directional antennas," in Proc. IEEE ICC'03, vol. 1, May 2003, pp. 348-352.

[2] S. Yi, Y. Pei, and S. Kalyanaraman, "On the capacity improvement of ad hoc wireless networks using directional antennas," in Proc. ACM MobiHoc'03, June 2003, pp. 108-116.

[3] I. Jawhar and J. Wu, "Resource allocation in wireless networks using directional antennas," in Proc. IEEE PERCOM'06, Mar. 2006.

[4] S. Aeron, S. Venkatesh, "Scaling laws and operation of wireless ad-hoc and sensor networks," in Proc. IEEE IWSSP'03, Sept./Oct. 2003, pp. $367-370$.

[5] J. Zhang and S. C. Liew, "Capacity improvement of wireless ad hoc networks with directional antennae," ACM Mobile Computing and Communications Review, 2006.

[6] T. Ueda, S. Tanaka, D. Saha, S. Roy, and S. Bandyopadhyay, "A rotational sector-based, receiver-oriented mechanism for location tracking and medium access control in ad hoc networks using directional antenna," in Proc. IFIP PWC'03, Sept. 2003.

[7] S. Bandyopadhyay, M. N. Pal, D. Saha, T. Ueda, K. Hasuike, and R. Pal, "Improving system performance of ad hoc wireless network with directional antenna," in Proc. IEEE ICC'03, vol. 2, May 2003, pp. 1146-1150.

[8] J.-L. Hsu and I. Rubin, "Performance analysis of directional random access scheme for multiple access mobile ad-hoc wireless networks," in Proc. IEEE MILCOM'05, vol. 1, Oct. 2005, pp. 45-51.

[9] M. Sharif and B. Hassibi, "On the capacity of mimo broadcast channels with partial side information," IEEE Trans. Inform. Theory, vol. 51, no. 2 , pp. 506-522, Feb. 2005

[10] C. Bettstetter, C. Hartmann, and C. Moser, "How does randomized beamforming improve the connectivity of ad hoc networks?" in Proc. IEEE ICC'05, vol. 5, May 2005, pp. 3380-3385.

[11] T. M. Cover and J. A. Thomas, Elements of Information Theory, 2nd Edition. New York: Wiley-Interscience, July 2006.

[12] X. Liu and R. Srikant, "An information-theoretic view of connectivity in wireless sensor networks," in Proc. IEEE SECON'04, Oct. 2004. 